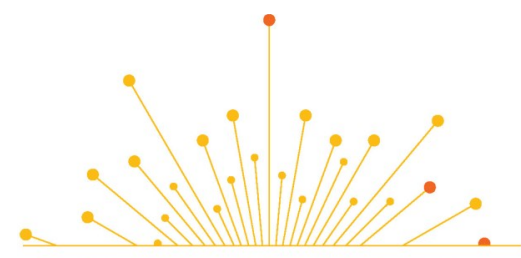

SCIENCE TABLE

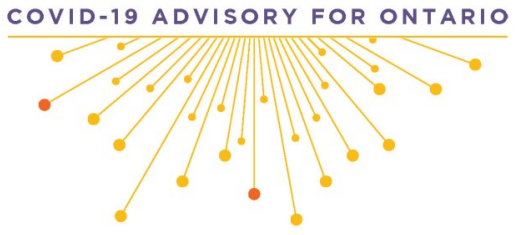

Version 1.1

Published: March 1, 2021

Updated on March 2, 2021. Version 1.0 is available under Additional Resources at https://doi.org/10.47326/ocsat.2021.02.11.1.0

Citation: Morris AM, Stall NM, Bobos P, et al. Tocilizumab for hospitalized patients with COVID-19. Science Briefs of the Ontario COVID-19 Science Advisory Table. 2021;2(11). https://doi.org/10.47326/ocsat.2021.02.11.1.0

Author Affiliations: The affiliations of the members of the Ontario COVID-19 Science Advisory Table can be found at https:// covid19-sciencetable.ca/.

Declarations of Interest: The declarations of interest of the members of the Ontario COVID-19 Science Advisory Table can be found at https://covid19-sciencetable.ca/.

About Us: The Ontario COVID-19 Science Advisory Table is a group of scientific experts and health system leaders who evaluate and report on emerging evidence relevant to the COVID-19 pandemic, to inform Ontario's response. Our mandate is to provide weekly summaries of relevant scientific evidence for the COVID-19 Health Coordination Table of the Province of Ontario, integrating information from existing scientific tables, Ontario's universities and agencies, and the best global evidence. The Science Table summarizes its findings for the Health Coordination Table and the public in Science Briefs.

The Drugs \& Biologics Clinical Practice Guidelines Working Group is a group of clinicians and scientists with recognized expertise in drugs, biologics, and clinical care. The Working Group will evaluate existing scientific data, disease epidemiology, drug availability, and implementation issues in order to develop Clinical Practice Guidelines for the treatment of COVID-19 using drugs and biologics. The Working Group reports its findings to the public and the Science Table. Its findings are also summarized in Science Briefs.

Correspondence to: Secretariat of the Ontario COVID-19 Science Advisory Table (info@covid19-sciencetable.ca)

\title{
Tocilizumab for Hospitalized Patients with COVID-19
}

Andrew M. Morris, Nathan M. Stall, Pavlos Bobos, Nicolas S. Bodmer, Stephanie Carlin, Elizabeth Leung, Antonina Maltsev, Katherine J. Miller, Laveena Munshi, Ayodele Odutayo, Fahad Razak, Sumit Raybardhan, Peter Jüni, Menaka Pai, on behalf of the Drugs \& Biologics Clinical Practice Guidelines Working Group and the Ontario COVID-19 Science Advisory Table

\section{Key Message}

Tocilizumab, an interleukin (IL-6) receptor antagonist, reduces the need for mechanical ventilation, progression to mechanical ventilation, and the combined endpoint of progression to mechanical ventilation or death in hospitalized patients with COVID-19, with no increase in serious adverse events.

Tocilizumab should be used in moderately ill hospitalized patients (i.e., requiring supplemental oxygen via nasal prongs) and critically ill hospitalized patients (i.e., requiring oxygen via venturi mask, high-flow nasal cannula, non-invasive mechanical ventilation, invasive mechanical ventilation, or extra-corporeal membrane oxygenation (ECMO)) with suspected or confirmed COVID-19 who are on recommended doses of dexamethasone (or another dose-equivalent corticosteroid) and are within 14 days of hospital admission or within 14 days of a new COVID-19 diagnosis if acquired in a healthcare setting.

Moderately ill patients should have additional evidence of systemic inflammation, defined as a C-reactive protein (CRP) of $75 \mathrm{mg} / \mathrm{L}$ or higher, and have evidence of disease progression (i.e., increasing oxygen or ventilatory requirements) while on dexamethasone or another dose-equivalent corticosteroid for treatment of COVID19.

\section{Lay Summary}

Tocilizumab Can Save Lives in Hospitalized COVID-19 Patients, with Moderate or Critical Illness

Patients with the most severe cases of COVID-19 develop dangerous inflammation that can compromise their breathing and damage their lungs, heart, kidney, and other vital organs.

As a COVID-19 treatment, tocilizumab should only be used in hospitalized patients whom doctors determine to be "moderately" to "critically" ill with COVID-19. Patients receiving tocilizumab should already be receiving corticosteroids (a class of drugs that also reduce inflammation) and supplemental oxygen. Tocilizumab should not be used in patients who have other bacterial, fungal or viral infections or active tuberculosis.

\section{How Tocilizumab Works}

Tocilizumab is a monoclonal antibody, already approved by Health Canada, that can reduce inflammation and improve survival in hospitalized patients with 
Copyright: 2021 Ontario COVID-19 Science Advisory Table. This is an open access document distributed under the terms of the Creative Commons Attribution License, which permits unrestricted use, distribution, and reproduction in any medium, provided that the original work is properly cited.

The views and findings expressed in this Science Brief are those of the authors and do not necessarily reflect the views of all of the members of the Ontario COVID-19 Science Advisory Table, its Working Groups, and its partners.
COVID-19 who are moderately to critically ill but not for those who are mildly ill.

Tocilizumab works by blocking the actions of IL-6, a chemical in our body that sends signals to increase the inflammatory response to infections. Tocilizumab is in a family of drugs called 'IL-6 inhibitors.'

\section{How We Came to Our Recommendations}

To understand tocilizumab's effect on moderately or critically ill patients hospitalized with COVID-19, we reviewed nine randomized controlled trials (RCTs) from around the world that compared this drug to placebo or to routine care alone). When their results were taken together, the studies found that tocilizumab can decrease death and decrease the chance that a COVID-19 patient will need invasive mechanical ventilation. One large trial also showed that tocilizumab decreases organ failure in COVID-19 as measured by the need for critical care support of the heart, lungs, and/or kidneys. Another large trial showed that tocilizumab decreases the length of stay in hospital for COVID-19 patients.

COVID-19 patients taking tocilizumab in these studies very rarely had reported side-effects; in fact, they were no more likely to suffer serious adverse events than patients in the placebo or routine care groups. Those few patients who did have side effects tended to experience other infections.

\section{Tocilizumab Availability in Ontario}

There is currently a very limited supply of tocilizumab in Ontario, and it is a lifesaving drug in other situations as well, including patient having life-threatening side effects to CAR-T therapy (a specialized therapy for some cancer patients). For that reason, it is not readily available at all Ontario hospitals. Moreover, the science currently does not allow us to predict which moderately or critically ill COVID-19 patients would most benefit from tocilizumab. All of this means we have to be very thoughtful in how to prescribe the drug for COVID-19 patients. We would like clinicians throughout the province to generate a good framework for using tocilizumab in COVID-19, based on their own experiences treating COVID-19 in their settings and guided by sound ethical principles.

\section{Our Recommendation for Using Tocilizumab as a Treatment for COVID-19}

We recommend that patients hospitalized with COVID-19 receive tocilizumab if they have moderate or severe illness requiring supplementary oxygen - whether it is low-flow oxygen through nasal prongs, higher-flow oxygen through external devices, or invasive mechanical ventilation with a breathing tube. Before patients receive tocilizumab, they should also be on recommended doses of dexamethasone (or a dose-equivalent corticosteroid), a drug that reduces inflammation. Patients should also be within 14 days of hospital admission (or within 14 days of a new COVID-19 diagnosis if the infection was acquired in a health care setting).

At this time, we do not recommend tocilizumab for patients who have been ill with COVID-19 for longer than these time periods, or for mildly ill patients. If patients are not on invasive mechanical ventilation, they should have other signs of inflammation: a lab test called "CRP" (or C-reactive protein) that is $75 \mathrm{mg} / \mathrm{L}$ or higher and breathing that is getting worse despite being on dexamethasone. 


\section{Summary}

\section{Background}

Some patients with progressive COVID-19 illness requiring hospitalization experience an excessive immune system response that results in severe organ injury. Corticosteroids have demonstrated a convincing mortality benefit in patients with COVID-19, supporting the concept that other anti-inflammatory agents may be effective as well. Tocilizumab is a Health Canada-approved monoclonal antibody that targets soluble and membrane-bound IL- 6 receptors, thereby blocking the actions of IL-6, an important inflammatory mediator. Tocilizumab is currently used in the treatment of cytokine release syndrome following chimeric antigen receptor Tcell (CAR-T) cancer therapy, refractory rheumatoid arthritis, and other rheumatologic conditions. As a monoclonal antibody with few existing indications and production by a single supplier, the Canadian supply of tocilizumab is limited.

\section{Questions}

Does tocilizumab improve patient outcomes such as mortality, need for mechanical ventilation, requirement for organ support, intensive care unit (ICU) length of stay, and hospital length of stay for individuals hospitalized with COVID-19?

Which hospitalized COVID-19 patients will receive the most benefit from tocilizumab?

\section{Findings}

As of February 21, 2021, nine randomized controlled trials (RCTs) have been released, either as preprints or in peer-reviewed journals, studying the effect of tocilizumab on a total of 5923 patients hospitalized with COVID-19. Patients with COVID-19 enrolled in these trials had illness severity ranging from moderately to critically ill.

Meta-analyses of RCTs demonstrate that tocilizumab reduces mortality when compared to control or usual care with a pooled risk ratio of 0.90 (95\% confidence interval [Cl] 0.83 to 0.97 ). In patients not on invasive mechanical ventilation (IMV) or extra-corporeal membrane oxygenation (ECMO) at baseline, tocilizumab decreases the progression to IMV with a risk ratio of 0.78 (95\% $\mathrm{Cl} 0.68$ to 0.90$)$. Tocilizumab also decreases the progression to death or IMV with a risk ratio of $0.83(95 \% \mathrm{Cl} 0.77$ to 0.90$)$, when compared to control.

Based on all available data, serious adverse events, as reported by investigators, are less common with tocilizumab than with control, with a risk ratio of $0.89(95 \% \mathrm{Cl}$ 0.74 to 1.07). The most common serious adverse events reported include infection, neutropenia, thrombocytopenia/bleeding, and hepatotoxicity.

Two RCTs included additional outcomes of relevance to clinicians and patients. The REMAP-CAP trial, which enrolled only patients within 24 hours of requiring respiratory or cardiovascular organ support showed that tocilizumab was associated with decreased requirement for organ support. The RECOVERY trial, which enrolled patients hospitalized with COVID-19 with a serum CRP of $75 \mathrm{mg} / \mathrm{L}$ or higher (indicating systemic inflammation), and a peripheral oxygen saturation $\left(\mathrm{SaO}_{2}\right)<92 \%$ on room air or requiring oxygen therapy (indicating hypoxia) demonstrated that tocilizumab was associated with a greater chance of discharge from hospital alive at 28-days. 


\section{Practical Considerations}

Tocilizumab should not be used in individuals with a demonstrated intolerance to tocilizumab, or who have evidence of active tuberculosis, bacterial, fungal, viral, or other infection. Patients on tocilizumab should be monitored for complications of this drug, including hepatotoxicity, thrombocytopenia, neutropenia, and latepresenting secondary infections. There is currently a limited supply of tocilizumab, and there are currently no evidence-based frameworks that would predict which moderately or critically ill patients would most benefit from tocilizumab; this makes evidence-based rationing challenging and introduces the possibility of significant inequity in distribution and administration. We encourage the development of local, regional, and provincial criteria for rationing of tocilizumab based on sound ethical principles to ensure that those most likely to experience clinical benefit can access it. Dosing of tocilizumab should also adhere to strategies that best conserve drug vials.

\section{Recommendation}

\section{Critically III Patients}

Tocilizumab (dosed according to body weight) is recommended for critically ill patients with suspected or confirmed COVID-19, who are on recommended doses of dexamethasone therapy (or a dose-equivalent corticosteroid) AND are within 14 days of hospital admission (or within 14 days of a new COVID-19 diagnosis if the infection was nosocomially acquired).

A second dose of tocilizumab may be considered after 24 hours if the patient is not improving.

The dose of intravenous tocilizumab may be determined by a weight-based dose strategy $(8 \mathrm{mg} / \mathrm{kg}$, maximum dose $800 \mathrm{mg}$ ) OR by a weight-based dose banding strategy ( $800 \mathrm{mg}$ if weight $>90 \mathrm{~kg} ; 600 \mathrm{mg}$ if weight $>65 \mathrm{and} \leq 90 \mathrm{~kg} ; 400 \mathrm{mg}$ if weight $>40$ and $\leq 65 \mathrm{~kg}$; and $8 \mathrm{mg} / \mathrm{kg}$ if weight $\leq 40 \mathrm{~kg}$ ).

\section{Moderately III Patients}

Tocilizumab (dosed according to body weight) is recommended for moderately ill patients with suspected or confirmed COVID-19, who have evidence of systemic inflammation, defined as a serum CRP of $75 \mathrm{mg} / \mathrm{L}$ or higher AND have evidence of disease progression (i.e., increasing oxygen or ventilatory requirements) despite 2448 hours of recommended doses of dexamethasone therapy (or a dose-equivalent corticosteroid) AND are within 14 days of hospital admission (or within 14 days of a new COVID-19 diagnosis if the infection was nosocomially acquired).

A second dose of tocilizumab may be considered after 24 hours if the patient is not improving, with dosing strategies being the same as for critically ill patients.

\section{Mildly III Patients}

Tocilizumab is not recommended outside of clinical trials for patients who are mildly ill with suspected or confirmed COVID-19.

Please see the Methods section below section below for a description of COVID-19 illness severity criteria.

\section{Background}

Infection with SARS-CoV-2, the virus causing COVID-19, involves a period of viral replication, usually with mild or no symptoms. In some patients with progressive disease requiring hospitalization, there is excessive and harmful activation of the 
immune system, marked by high levels of circulating inflammatory markers including IL-1, IL-6, tumor necrosis factor (TNF)-a, D-dimers, ferritin, and CRP. This vigorous immune response can result in organ damage, with diffuse inflammation of lung alveoli, neutrophilic infiltrates, and microvascular thrombosis. This aberrant hyperactivation of the immune system is an important therapeutic target in COVID19. Corticosteroids, including dexamethasone, have already demonstrated convincing beneficial evidence in COVID-19, supporting the concept that antiinflammatory therapy is a reasonable target of therapy. ${ }^{1,2}$

The drugs tocilizumab and sarilumab are Health Canada-approved monoclonal antibodies that target soluble and membrane-bound IL-6 receptors, thereby blocking the actions of IL-6. Tocilizumab is the standard of care when treating patients with cytokine release syndrome following chimeric antigen receptor T-cell (CAR-T) cancer therapy (presently used in the treatment of diffuse large B-cell lymphoma and acute lymphoblastic leukemia); sarilumab is a less studied alternative. ${ }^{3}$ Tocilizumab is also used in refractory rheumatoid arthritis, and other rheumatologic conditions.

Because it is a monoclonal antibody with few existing indications and is manufactured by a single producer, Canadian supply of tocilizumab is extremely limited. In Ontario, it is estimated that $8000 \mathrm{mg}$ of tocilizumab-the equivalent of roughly 10 doses-is used monthly outside of COVID-19 therapy, mainly for complications of CAR-T therapy. Regulations mandate that hospitals have protected stock of 4 doses $(8 \mathrm{mg} / \mathrm{kg}$ ) of tocilizumab for every patient undergoing 10-day CAR-T therapy.

\section{Questions}

Does tocilizumab improve patient outcomes such as mortality, need for mechanical ventilation, requirement for organ support, intensive care unit (ICU) length of stay, and hospital length of stay for individuals hospitalized with COVID-19?

Which hospitalized COVID-19 patients will receive the most benefit from tocilizumab?

\section{Findings}

As of February 20, 2021, nine randomized controlled trials (RCTs) have been released, either as preprints or in peer-reviewed journals, studying the effect of tocilizumab on a total of 5923 patients hospitalized with COVID-19. ${ }^{4-12}$ There is heterogeneity in enrolled patients, ranging from moderately ill patients on supplemental oxygen via nasal prongs, non-invasive mechanical ventilation (NIMV, high flow nasal cannula (HFNC), or continuous positive airway pressure (CPAP)), to critically ill patients receiving IMV. Patients in the RECOVERY trial were required to have biochemical evidence of inflammation with an increased serum C-reactive protein (CRP) of $75 \mathrm{mg} / \mathrm{L}$ or higher. ${ }^{12}$

Of the 9 RCTs, 3 were placebo-controlled and randomized patients to tocilizumab or placebo and standard of care in a 2:1 ratio., ${ }^{5,711}$ The other 6 RCTs were compared to standard-of-care alone, with 1:1 randomization to tocilizumab or standard-ofcare. ${ }^{4,6,8-10,12}$ One trial, REMAP-CAP, randomized 48 patients to sarilumab as an alternative to tocilizumab. ${ }^{9}$

Unlike the other trials, REMAP-CAP $(n=895)$ was limited to critically ill patients within 24 hours of requiring ventilatory support ranging from HFNC to IMV. 


\section{Outcome: Overall Mortality}

Tocilizumab reduces overall mortality in hospitalized patients with moderate or severe COVID-19 illness when compared to control (placebo and standard of care, or standard of care alone). The 9 RCTs with available data on mortality enrolled a total of 5923 patients. ${ }^{4-12}$ In a meta-analysis, the pooled risk ratio is $0.90(95 \% \mathrm{Cl} 0.83$ to 0.97), favouring tocilizumab; the effect is dominated by the large number of participants in the RECOVERY trial. The results of the meta-analysis are shown in Figure 1.

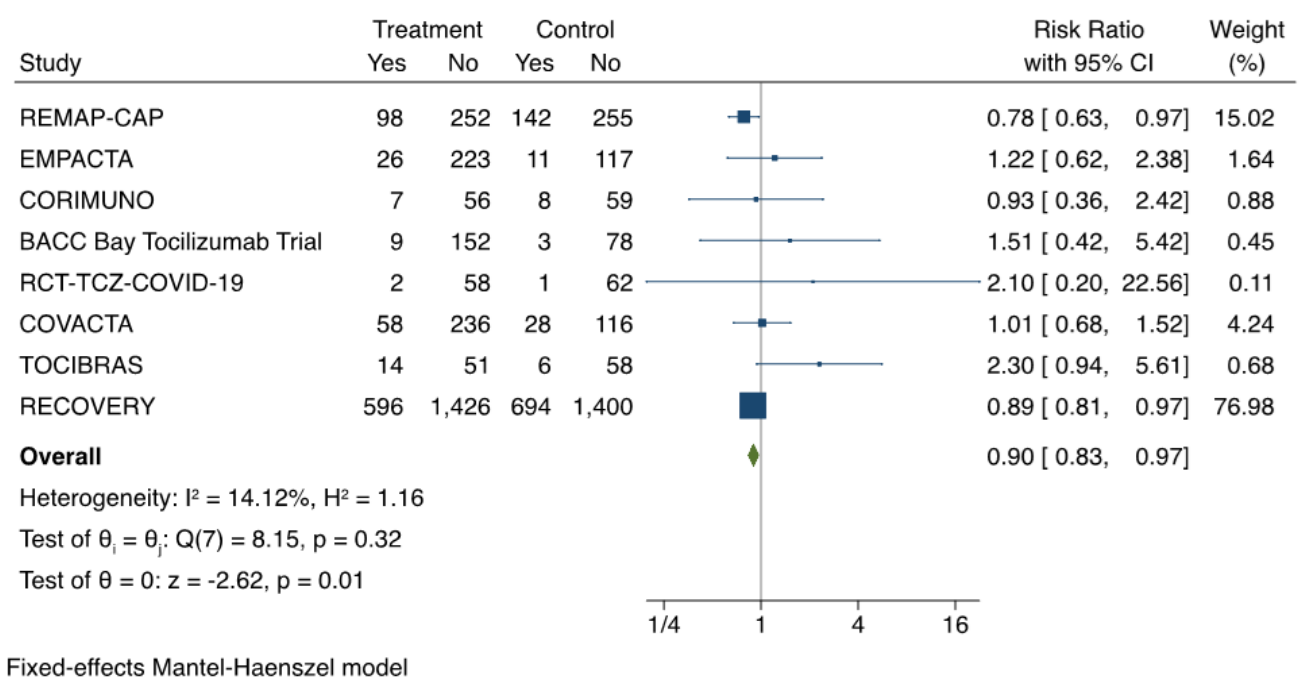

Figure 1. Meta-Analysis of the Effect of Tocilizumab vs. Control on All-Cause Mortality in Randomized Controlled Trials of Patients Hospitalized with Moderate or Severe COVID-19 IIIness

Forest plot presenting the meta-analyzed effect of tocilizumab vs. control on all-cause mortality in RCTs of patients hospitalized with moderate or severe COVID-19 illness. Each square presents the results of an individual RCT with the size of the square being proportional to the weights used in the meta-analysis and the horizontal lines indicating the 95\% confidence intervals. The solid vertical line represents no effect on mortality and the solid diamond indicates the overall summary estimate.

\section{Outcome: Invasive Mechanical Ventilation}

When looking at patients not on IMV or ECMO at baseline, tocilizumab decreases the progression to IMV. The results of the meta-analysis from 6 RCTs are shown in Figure $2 .^{5-8,11,12}$ The pooled risk ratio of progression to IMV associated with tocilizumab is 0.78 ( $95 \% \mathrm{Cl} 0.68$ to 0.90$)$.

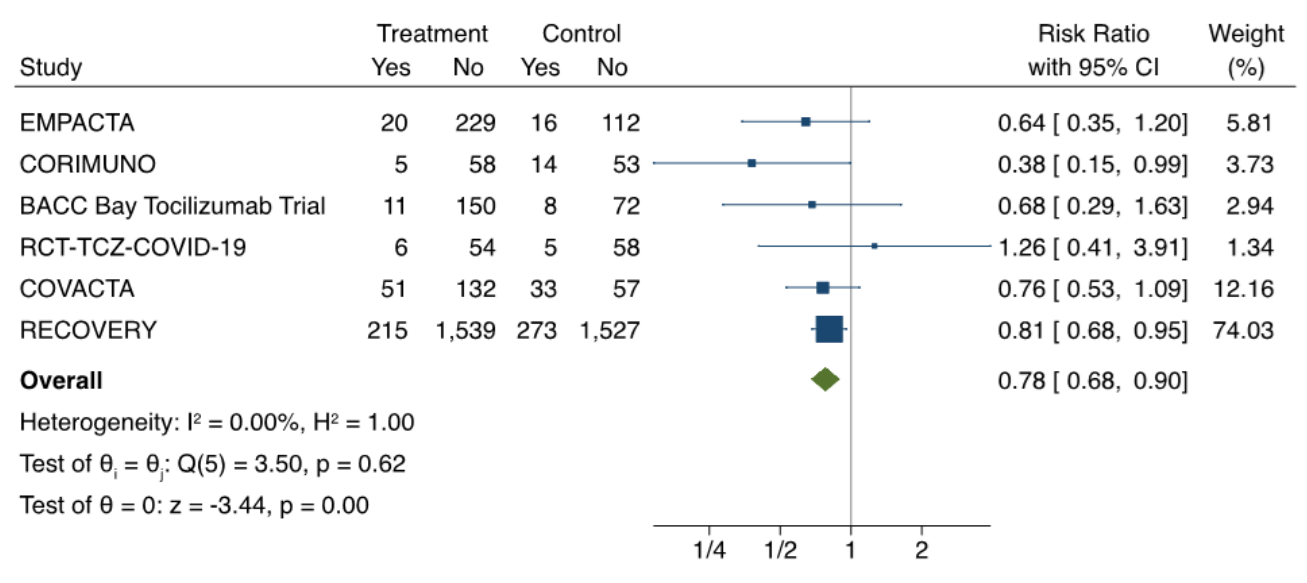

Fixed-effects Mantel-Haenszel model

Figure 2. Meta-Analysis of the Effect of Tocilizumab vs. Control on Progression to Mechanical Ventilation in Randomized Controlled Trials of Patients Hospitalized with Moderate or Severe COVID-19 Illness

Forest plot presenting the meta-analyzed effect of tocilizumab vs. control on progression to IMV in RCTs of patients 
hospitalized with moderate or severe COVID-19 illness. Each square presents the results of an individual RCT with the size of the square being proportional to the weights used in the meta-analysis and the horizontal lines indicating the 95\% confidence intervals. The solid vertical line represents no effect on IMV and the solid diamond indicates the overall summary estimate.

\section{Outcome: Composite of Mortality or Invasive Mechanical Ventilation}

Because patients who die may not proceed to IMV before death, it is important to look at the composite outcome of mortality or IMV. The results of the meta-analysis from 5 RCTs are presented in Figure 3.,.$^{5,-9,12}$ Tocilizumab is associated with a pooled risk ratio of $0.83(95 \% \mathrm{Cl} 0.77$ to 0.90$)$ of mortality or progression to IMV when compared to control.

\begin{tabular}{|c|c|c|c|c|c|c|c|}
\hline \multirow[b]{2}{*}{ Study } & \multicolumn{2}{|c|}{ Treatment } & \multicolumn{2}{|c|}{ Control } & & \multirow{2}{*}{$\begin{array}{l}\text { Risk Ratio } \\
\text { with } 95 \% \mathrm{Cl}\end{array}$} & \multirow{2}{*}{$\begin{array}{c}\text { Weight } \\
(\%)\end{array}$} \\
\hline & Yes & No & Yes & No & & & \\
\hline REMAP-CAP & 100 & 142 & 144 & 129 & $\longrightarrow$ & $0.78[0.65,0.94]$ & 15.43 \\
\hline EMPACTA & 30 & 219 & 25 & 103 & $\because$ & $0.62[0.38,1.00]$ & 3.76 \\
\hline CORIMUNO & 11 & 52 & 18 & 49 & & $0.65[0.33,1.27]$ & 1.99 \\
\hline BACC Bay Tocilizumab Trial & 17 & 144 & 10 & 70 & 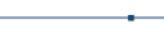 & $-0.84[0.41,1.76]$ & 1.52 \\
\hline RECOVERY & 571 & 1,183 & 687 & 1,113 & 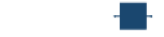 & $0.85[0.78,0.93]$ & 77.30 \\
\hline Overall & & & & & & $0.83[0.77,0.90]$ & \\
\hline \multicolumn{8}{|c|}{ Heterogeneity: $\mathrm{I}^{2}=0.00 \%, \mathrm{H}^{2}=1.00$} \\
\hline \multicolumn{8}{|c|}{ Test of $\theta_{i}=\theta_{j}: Q(4)=2.67, p=0.61$} \\
\hline \multicolumn{8}{|l|}{ Test of $\theta=0: z=-4.66, p=0.00$} \\
\hline & & & & & $1 / 2$ & & \\
\hline
\end{tabular}

Figure 3. Meta-Analysis of the Effect of Tocilizumab vs. Control on the Composite Outcome of Mortality or Progression to Invasive Mechanical Ventilation in Randomized Control Trials of Patients Hospitalized with Moderate or Severe COVID-19 Illness

Forest plot presenting the meta-analyzed effect of tocilizumab vs. control on the composite outcome of mortality or progression to IMV in RCTs of patients hospitalized with moderate or severe COVID-19 illness. Each square presents the results of an individual RCT with the size of the square being proportional to the weights used in the metaanalysis and the horizontal lines indicating the 95\% confidence intervals. The solid vertical line represents no effect on mortality or IMV and the solid diamond indicates the overall summary estimate.

\section{Adverse Events}

Based on all available data, serious adverse events, as reported by investigators, are less common with tocilizumab than with control (placebo and standard of care, or standard of care alone), with a risk ratio of 0.89 (95\% Cl 0.74 to 1.07). The most common adverse events reported in the RCTs included infection, neutropenia, thrombocytopenia/bleeding, and hepatotoxicity. Prespecified safety outcomes from RECOVERY included cause-specific mortality and major cardiac arrhythmia; infection was not specifically investigated. There were three reports of serious adverse reactions believed to be related to tocilizumab in RECOVERY: one each of otitis externa, Staphylococcus aureus bacteraemia, and lung abscess, all of which resolved with standard treatment. Preliminary information on cause-specific mortality from the RECOVERY trial shows no evidence of excess deaths from other infections, but further information is expected in the peer-reviewed publication. 


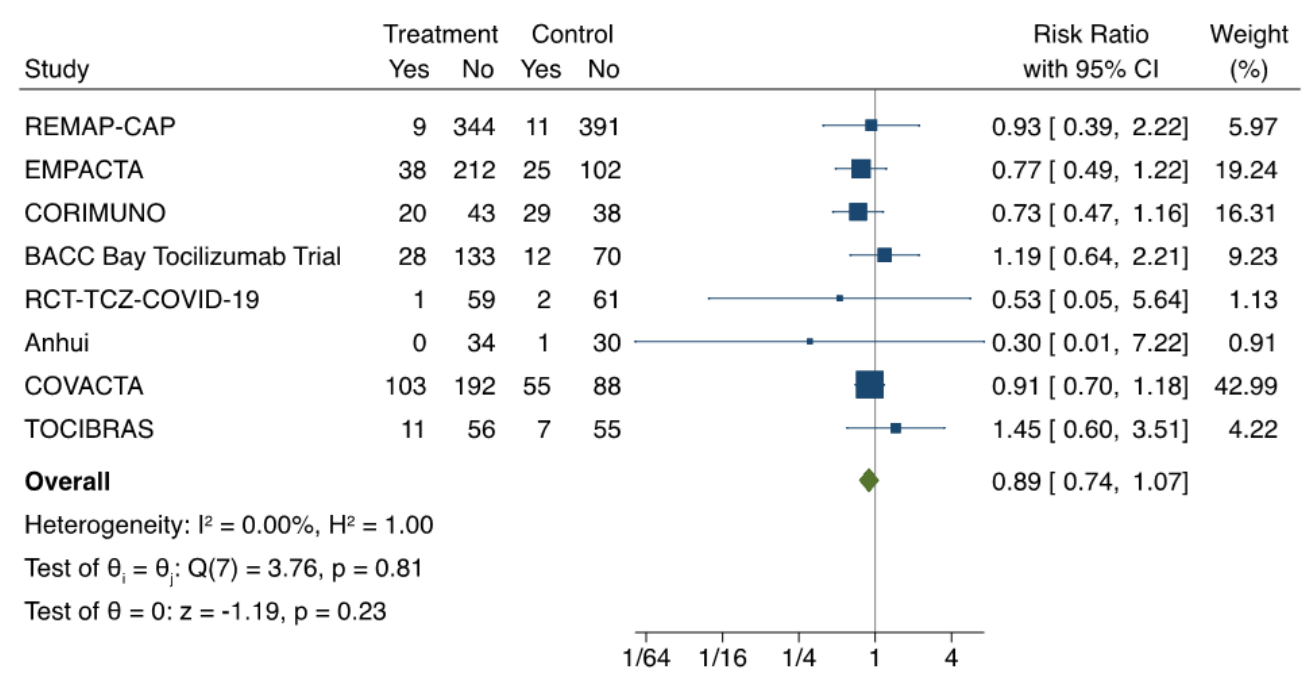

Fixed-effects Mantel-Haenszel model

Figure 4. Meta-Analysis of Serious Adverse Events of Tocilizumab vs. Control in Randomized Controlled Trials of Patients Hospitalized with Moderate or Severe COVID-19 Illness

Forest plot presenting the meta-analyzed effect of tocilizumab vs. control on serious adverse events in RCTs of patients hospitalized with moderate or severe COVID-19 illness. Each square presents the results of an individual RCT with the size of the square being proportional to the weights used in the meta-analysis and the horizontal lines indicating the $95 \%$ confidence intervals. The solid vertical line represents no effect on serious adverse events and the solid diamond indicates the overall summary estimate.

\section{Other Outcomes: Organ Support, Hospital Length of Stay}

Two RCTs included additional outcomes of relevance to clinicians and patients. REMAP-CAP is an international, multicentre randomized controlled trial using an adaptive design, which enrolled patients with severe community-acquired pneumonia. ${ }^{8}$ Eligible patients had PCR-confirmed or suspected COVID-19 within 24 hours of requiring respiratory or cardiovascular organ support, as indicated by ventilatory support via HFNC ( $>30 \mathrm{~L} / \mathrm{min}$ with a fraction of inspired oxygen (FiO2) $>0.40$ ), non-invasive or invasive mechanical ventilation, or circulatory support with vasopressors or inotropes. One of its outcomes was respiratory and cardiovascular organ support-free days up to day 21 from trial enrollment. In this outcome, a higher number is better, indicating less need for organ support. To account for death, all hospital deaths were assigned an organ support-free day of -1 ; a participant alive on organ support for the full 21 days would otherwise have an OSFD of 0 . In 353 subjects receiving tocilizumab, the median of organ support-free days was 10 , whereas it was 0 in the 402 subjects receiving standard therapy. The adjusted odds ratio of organ support-free days was 1.64 (95\% Cl 1.25 to 2.14), favouring tocilizumab. An odds ratio greater than 1 represents improved survival, more organ support-free days, or both. The probability of tocilizumab being superior to control was $>99.9 \%$. A similar analysis was performed for the 48 patients receiving sarilumab, with a median of organ support-free days of 11 days. The odds ratio of organ support-free days was 1.76 (95\% $\mathrm{Cl} 1.17$ to 2.91$)$, favouring sarilumab. The probability of sarilumab being superior to control was $>99.5 \%$.

RECOVERY is a UK-based randomized, controlled, open label platform trial for patients with moderate or severe COVID-19 illness. ${ }^{12}$ It enrolled patients with a serum CRP of $75 \mathrm{mg} / \mathrm{L}$ or higher (indicating systemic inflammation) and a peripheral oxygen saturation $\left(\mathrm{SaO}_{2}\right)<92 \%$ on room air or requiring oxygen therapy (indicating hypoxia). Patients were randomized to usual standard of care or usual standard of care plus a weight-based banded dose of tocilizumab. A second dose of tocilizumab could be administered $12-25$ hours later if there was no improvement. The primary outcome of RECOVERY was 28-day mortality. However, a secondary outcome was 
discharge from hospital alive at 28-days. Allocation to tocilizumab was associated with a greater probability of discharge from hospital alive within 28 days ( $54 \%$ vs. $47 \%$; rate ratio $1.22,95 \% \mathrm{Cl} 1.12$ to 1.34 ).

\section{Interpretation}

In patients hospitalized with moderate or severe COVID-19 illness requiring at least supplemental oxygen, tocilizumab reduces mortality and reduces the need for IMV. It also reduces the combined end-point of IMV and mortality. The effect of tocilizumab is consistent across subgroups of moderately or critically ill patients with COVID-19.

There are a number of practical considerations surrounding the use of tocilizumab in patients hospitalized with COVID-19. Based on the enrollment criteria for the available RCTs, and the known side effects of this drug, it should not be used in individuals with a demonstrated intolerance to tocilizumab, or evidence of active tuberculosis, bacterial, fungal, viral, or other infection (this includes patients who have active non-COVID-19 infection, in addition to COVID-19). Patients on tocilizumab should be monitored for complications of this drug, including hepatotoxicity, thrombocytopenia, neutropenia, and late-presenting secondary infections (including reactivation of latent tuberculosis infection, invasive fungal infections, and opportunistic bacterial or viral infections). There is currently no specific data to guide clinicians in monitoring for complications of tocilizumab in the setting of COVID-19. However, extrapolating from the use of tocilizumab in other approved settings (e.g., rheumatoid arthritis), it would be reasonable to monitor liver enzymes and a complete blood count, and signs and symptoms of infection during hospitalization and for 4 weeks after tocilizumab administration.

In Ontario, tocilizumab is currently in limited supply. This has the potential to contribute to inequity across the province, where not all patients have the same opportunity to access this drug. At this time, we are not able to recommend an evidence-based framework that would predict which patients (among those encompassed by our recommendations below) would most benefit from tocilizumab. Until further evidence of subgroup benefits is available, we encourage the development of local, regional, and provincial criteria for rationing of this drug, based on sound ethical principles, to ensure that those most likely to experience clinical benefit can access the drug. ${ }^{14}$

To ensure evidence-based stewardship of tocilizumab, we suggest the following strategies:

Selection of dosing strategy: the RECOVERY trial used a weight-based dose banding strategy, while the REMAP-CAP trial used a weight-based dose strategy. Both appear acceptable. Clinicians are encouraged to use the dosing strategy that would best conserve drug vials and should consult a pharmacist when doing so; depending on the patient's weight, one or the other dosing strategy will yield a lower dose.

Corticosteroid use: tocilizumab use should be restricted to patients already on recommended doses of dexamethasone (or a dose-equivalent corticosteroid). This reflects the high concomitant use of dexamethasone in REMAP-CAP, RECOVERY, and other recent RCTs of tocilizumab.

CRP level: RECOVERY required a serum CRP level of $75 \mathrm{mg} / \mathrm{L}$ or higher. Because it clearly showed a benefit for patients moderately ill with COVID-19, this cut-off will assist with tocilizumab stewardship in those moderately ill with COVID-19.

Limits on timing of introduction: tocilizumab should be restricted to patients who 
are within 14 days of hospital admission (or within 14 days of a new COVID-19 diagnosis if the infection was nosocomially acquired).

Limits on second dosing: a second dose of tocilizumab may be considered if patients do not have improved ventilatory status after 24 hours, as per the RECOVERY trial protocol. However, there is a paucity of data supporting administration of a second dose, and routine second dosing will exacerbate drug shortages. We suggest administering a second dose only if there is objective evidence of non-improvement (e.g., increasing oxygen or ventilatory requirements), and in accordance with local, regional, and/or provincial ethical frameworks.

\section{Recommendations}

\section{Critically III Patients}

Tocilizumab (dosed according to body weight) is recommended for critically ill patients with suspected or confirmed COVID-19, who are: on a recommended dose of dexamethasone therapy (or a dose-equivalent corticosteroid); AND are within 14 days of hospital admission (or within 14 days of a new COVID-19 diagnosis if nosocomially acquired).

A second dose of tocilizumab may be considered after 24 hours if the patient is not improving.

The dose of intravenous tocilizumab may be determined by a weight-based dose strategy $(8 \mathrm{mg} / \mathrm{kg}$, maximum dose $800 \mathrm{mg}$ ) OR by a weight-based dose banding strategy ( $800 \mathrm{mg}$ if weight $>90 \mathrm{~kg} ; 600 \mathrm{mg}$ if weight $>65$ and $\leq 90 \mathrm{~kg} ; 400 \mathrm{mg}$ if weight $>40$ and $\leq 65 \mathrm{~kg}$; and $8 \mathrm{mg} / \mathrm{kg}$ if weight $\leq 40 \mathrm{~kg}$ ).

\section{Moderately III Patients}

Tocilizumab (dosed according to body weight) is recommended for moderately ill patients with suspected or confirmed COVID-19, who have evidence of systemic inflammation, defined as a serum CRP of $75 \mathrm{mg} / \mathrm{L}$ or higher AND have evidence of disease progression (i.e., increasing oxygen or ventilatory requirements) despite 2448 hours of a recommended dose of dexamethasone therapy (or a dose-equivalent corticosteroid) AND are within 14 days of hospital admission (or within 14 days of a new COVID-19 diagnosis if the infection was nosocomially acquired).

A second dose of tocilizumab may be considered after 24 hours if the patient is not improving.

The dose of intravenous tocilizumab may be determined by a weight-based dose strategy $(8 \mathrm{mg} / \mathrm{kg}$, maximum dose $800 \mathrm{mg}$ ) OR by a weight-based dose banding strategy ( $800 \mathrm{mg}$ if weight $>90 \mathrm{~kg} ; 600 \mathrm{mg}$ if weight $>65$ and $\leq 90 \mathrm{~kg} ; 400 \mathrm{mg}$ if weight $>40$ and $\leq 65 \mathrm{~kg}$; and $8 \mathrm{mg} / \mathrm{kg}$ if weight $\leq 40 \mathrm{~kg}$ ).

\section{Mildly III Patients}

Tocilizumab is not recommended outside of clinical trials for patients who are mildly ill with suspected or confirmed COVID-19.

Please see the Methods section for a description of COVID-19 illness severity criteria.

\section{Methods Used for This Science Brief}

We searched PubMed, Google Scholar, the COVID-19 Rapid Evidence Reviews, the Joanna Briggs Institute's COVID-19 Special Collection, LitCovid in PubMed, the 
Oxford COVID-19 Evidence Service, the World Health Organization's Global Literature on Coronavirus Disease, and other COVID-19 specific resources listed by the Guidelines International Network and the McMaster Health Forum. In addition, we retrieved reports citing relevant articles through Google Scholar and reviewed references from identified articles for additional studies. The search was last updated on February 21, 2021. We conducted our meta-analyses using STATA Release 16.0 software (StataCorp LLC, College Station, TX) and used a MantelHaenszel fixed-effects meta-analysis to combine outcomes (overall mortality, invasive mechanical ventilation, composite of mortality or invasive mechanical ventilation and serious adverse events) from individual RCTs and pooled risk ratios and $95 \% \mathrm{Cls}$.

For therapeutic recommendations, we used the following definitions for severity:

\section{Critically III}

Patients requiring ventilatory and/or circulatory support; also includes patients requiring high-flow nasal cannula, non-invasive ventilation, or higher concentrations of oxygen by mask. These patients are usually managed in intensive care units but may be managed on other inpatient units.

\section{Moderately III}

Patients newly requiring low-flow supplemental oxygen to keep an oxygen saturation of at least $92 \%$. These patients are usually managed on hospital wards.

\section{Mildly III}

Patients who do not require new or additional supplemental oxygen from their baseline status, intravenous fluids, or other physiological support. These patients are usually managed in an ambulatory/outpatient setting.

\section{Author Contributions}

AMM wrote the first draft of the Science Brief. MP, PB, NB, AO and PJ performed the data synthesis and meta-analysis. All authors contributed to the conception of the Science Brief, revised it critically for important intellectual content, and approved the final version.

The authors would like to thank all members of the Drugs and Biologics Clinical Practice Guideline Working Group for their contribution to this Science Brief and the Guidelines currently available at antimicrobialstewardship.com and https://covid19sciencetable.ca/.

\section{Acknowledgements}

The authors would like to acknowledge the important contributions of the Ontario COVID-19 Antimicrobial and Immunomodulatory Clinical Practice Guidelines Committee throughout the COVID-19 pandemic. Those members include: Karim Ali, MD, Niagara Health System; Amir Amiri, Patient Partner; Nisha Andany, MD MPH, Sunnybrook Health Sciences Centre; Sally Bean, Ethicist, Sunnybrook Health Sciences Centre; Zain Chagla, MD, Hamilton Health Sciences; Bill Ciccotelli, MD, Grand River Hospital ; Pavani Das, MD, North York General Hospital; Linda Dresser, PharmD, University Health Network; Sameer Elsayed, MD, LHSC Victoria Hospital; Wayne Gold, MD, University Health Network; Kevin Gough, MD, St. Michael's Hospital, Unity Health Toronto; Chris Graham, MD, Trillium Health Partners; Rebecca Greenberg, Ethicist, Sinai Health; Shahid Husain, MD MS, University Health Network; 
Neal Irfan, PharmD BScPhm, Hamilton Health Sciences; Susan John, MD, Scarborough Health Network; Rupert Kaul, MD PhD, University Health Network; Elizabeth Leung, PharmD MSCl, St. Michael's Hospital, Unity Health Toronto; Brian Minnema, MD, St. Joseph's Hospital, Unity Health Toronto; Jeya Nadarajah, MD, MSc, Markham Stouffville Hospital; Caroline Nott, MD, The Ottawa Hospital; Lesley Palmay, BSc BScPhm MSc, Sunnybrook Health Sciences Centre; Alexandra Persichino, BScPhm, Thunder Bay Regional Health Sciences Center; Sumit Raybardhan, MPH, North York General Hospital; Kathryn Timberlake, PharmD, Hospital for Sick Children; Liliana Volnikova, Patient Partner; Anupma Wadhwa, MD MEd, Hospital for Sick Children; Evan Wilson, MD MSc, Kingston Health Sciences Centre; Peter Wu, MD MSc, University Health Network; Ivan Ying, MD, Mackenzie Health.

\section{References}

1. Jüni $P$, Odutayo $A$, Allen $U$, et al. Dexamethasone in patients hospitalized for COVID-19. Sci Briefs Ont COVID-19 Sci Advis Table. 2020;1(1). https:// doi.org/10.47326/ocsat.2020.01.01.1.0

2. Sterne JAC, Murthy S, Diaz JV, et al. Association Between Administration of Systemic Corticosteroids and Mortality Among Critically III Patients With COVID19: A Meta-analysis. JAMA. Published online September 2, 2020. https:// doi.org/10.1001/jama.2020.17023

3. Shimabukuro-Vornhagen $A$, Gödel $P$, Subklewe $M$, et al. Cytokine release syndrome. J Immunother Cancer. 2018;6(1):56. https://doi.org/10.1186/s40425018-0343-9

4. Veiga VC, Prats JAGG, Farias DLC, et al. Effect of tocilizumab on clinical outcomes at 15 days in patients with severe or critical coronavirus disease 2019: randomised controlled trial. BMJ. 2021;372:n84. https://doi.org/10.1136/ bmj.n84

5. Salama C, Han J, Yau L, et al. Tocilizumab in Patients Hospitalized with Covid-19 Pneumonia. N Engl J Med. 2021;384(1):20-30. https://doi.org/10.1056/ NEJMoa2030340

6. Salvarani C, Dolci G, Massari M, et al. Effect of Tocilizumab vs Standard Care on Clinical Worsening in Patients Hospitalized With COVID-19 Pneumonia: A Randomized Clinical Trial. JAMA Intern Med. 2021;181(1):24. https:// doi.org/10.1001/jamainternmed.2020.6615

7. Stone JH, Frigault MJ, Serling-Boyd NJ, et al. Efficacy of Tocilizumab in Patients Hospitalized with Covid-19. N Engl J Med. 2020;383(24):2333-2344. https:// doi.org/10.1056/NEJMoa2028836

8. Hermine $\mathrm{O}$, Mariette $\mathrm{X}$, Tharaux $\mathrm{P}-\mathrm{L}$, et al. Effect of Tocilizumab vs Usual Care in Adults Hospitalized With COVID-19 and Moderate or Severe Pneumonia: A Randomized Clinical Trial. JAMA Intern Med. 2020;181(1):32. https:// doi.org/10.1001/jamainternmed.2020.6820

9. REMAP-CAP Investigators, Gordon AC, Mouncey PR, et al. Interleukin-6 Receptor Antagonists in Critically III Patients with Covid-19. N Engl J Med. 2021. Published online February 25, 2021. https://doi.org/10.1056/NEJMoa2100433

10. Wang D, Fu B, Peng Z, et al. Tocilizumab Ameliorates the Hypoxia in COVID-19 Moderate Patients with Bilateral Pulmonary Lesions: A Randomized, Controlled, Open-Label, Multicenter Trial. Social Science Research Network; 2020. https:// 
doi.org/10.2139/ssrn.3667681

11. Rosas IO, Brau N, Waters M, et al. Tocilizumab in Hospitalized Patients with Severe Covid-19 Pneumonia. N Engl J Med. 2021. Published online February 25, 2021. https://doi.org/ 10.1056/NEJMoa2028700

12. Horby PW, Pessoa-Amorim G, Peto L, et al. Tocilizumab in patients admitted to hospital with COVID-19 (RECOVERY): preliminary results of a randomised, controlled, open-label, platform trial. medRxiv. Published online February 11, 2021:2021.02.11.21249258. https://doi.org/10.1101/2021.02.11.21249258

13. Soriano MC, Vaquero $C$, Ortiz-Fernández A, Caballero A, Blandino-Ortiz A, de Pablo R. Low incidence of co-infection, but high incidence of ICU-acquired infections in critically ill patients with COVID-19. J Infect. 2021;82(2):e20-e21. https://doi.org/10.1016/j.jinf.2020.09.010

14. Emanuel EJ, Persad G, Upshur R, et al. Fair Allocation of Scarce Medical Resources in the Time of Covid-19. N Engl J Med. 2020;382(21):2049-2055. https://doi.org/10.1056/NEJMsb2005114 